\title{
Atomic processes in planetary nebulae
}

\author{
Xiaowei Liu \\ Kavli Institute for Astronomy and Astrophysics \\ and \\ Department of Astronomy, School of Physics, \\ Peking University, Beijing 100871, P. R. China \\ email: x.liu@pku.edu.cn
}

\begin{abstract}
Progress in the study of the atomic processes in planetary nebulae (PNe) is reviewed, focusing on the literature published since the last IAU symposium on PNe five years ago. High quality photoionization cross sections and recombination coefficients are now available for the first six ions of the trans-iron elements selenium and krypton, enabling robust modeling of their ionization structure and consequently converting the measured ionic abundances to elemental abundances. Major progress has been achieved in utilizing the recombination spectra of helium and heavy element ions to probe the nebular physical conditions. New ab initio, densitydependent effective recombination coefficients have been calculated for the recombination spectra of $\mathrm{O}$ II and N II, down to very low temperatures ( 100-300 K). Plasma diagnostics based entirely on those heavy element recombination lines are developed and applied to the observations. It is shown that these heavy element recombination lines originate predominately from cold plasmas of temperatures $\sim 1,000 \mathrm{~K}$, in agreement with the predictions of the bi-abundance model that has been proposed to explain the dichotomy of nebular plasma diagnostics and abundance determinations using collisional excited lines (CELs) on the one hand and optical recombination lines (ORLs) on the other.
\end{abstract}

Keywords. Atomic processes, atomic data, planetary nebulae: general

\section{Introduction}

Planetary nebulae (PNe) are low density plasmas ionized and heated by diluted UV radiation fields emitted by pr-white dwarfs. The plasmas are far from (local) thermodynamic equilibrium. Knowledge of a variety of relevant microscopic physical processes and their rate coefficients, e.g. photoionization and (radiative and di-electronic) recombination, charge exchange reactions, collisional excitation and de-excitation, and radiative processes, are required to understand the ionization and thermal structure of $\mathrm{PNe}$ and interpret their spectra. This contribution reviews progress on atomic processes in PNe achieved since the last IAU symposium on PNe five years ago, concentrating on plasma diagnostics newly developed. More comprehensive reviews on the atomic processes and the tools to calculate the relevant rate coefficients are reviewed respectively by Nahar (2003) and Bautista (2006) in the previous proceedings of this series of conferences. Given the large number of publications, it is infeasible to include and cite all the relevant papers in this limited space. References compiled when preparing this contribution are listed in the viewgraphs of the oral presentation, available online (http://astroatom.wordpress.com/2011/07/31/atomic-processes-in-photoionized-gaseous -nebulae/; thanks to Dr. C. Mendoza for this). The lists are bound to be incomplete. 


\section{Collisional excited lines (CELs)}

In the optical, the $[\mathrm{O}$ II] and $[\mathrm{S} \mathrm{II}]$ nebular line ratios are the most widely used densitydiagnostics. New collision strengths and transition probabilities are presented by Chen, Qing \& Li (2007) and Tayal (2007) for [O II] and by Tayal \& Zatsarinny (2010) for [S II], taking into account more configurations and levels than in previous calculations. The results compare well with the early calculations and confirm that relativistic effects are insignificant amongst the ground electron configuration of $[\mathrm{O} \mathrm{II}]$ and $[\mathrm{S} \mathrm{II}]$.

For temperature measurements, the most important diagnostics are provided by the [O III] and [N II] nebular to auroral line ratios. In a detailed study of the thermal structure of the most metal-poor blue compact galaxy I Zw 18, Péquignot (2008) compares collision strengths of $[\mathrm{O} \mathrm{III}]$ published in the literature. The comparison suggests that the data are in good shape and probably accurate to 4 and 2 per cent for the ${ }^{3} \mathrm{P}-{ }^{1} \mathrm{D}$ and ${ }^{3} \mathrm{P}$ $-{ }^{1} \mathrm{~S}$ transitions, respectively, for a wide range of temperature from 5,000-30,000 K. Larger scatters are seen in the collision strengths of the ${ }^{1} \mathrm{D}-{ }^{1} \mathrm{~S}$ transition, at the levels of $\sim 10$ and 6 per cent at $T_{\mathrm{e}}=10,000$ and $20,000 \mathrm{~K}$, respectively. The situation is less clear for [N II]. The new 58-LSJ state calculation of Tayal (2011) yields collision strengths amongst the fine-structure levels of the ground ${ }^{3} \mathrm{P}$ term that differ significantly from the earlier LS-coupling calculations.

Prominent [Fe III] lines are observed in bright $\mathrm{H}$ II regions such as the Orion Nebula and in some PNe, notably Mz 3, M 2-24 and the Eskimo Nebula (NGC 2392). The wide range of critical densities spanned by [Fe III] lines makes them particularly useful to probe high density regions embedded in diffuse nebulae (Zhang \& Liu 2002, 2003; Zhang et al., this volume). Large-scale calculations of atomic data (collision strengths and radiative transition rates) and a non-LTE spectral model for [Fe III] are presented by Bautista, Ballance \& Quinet (2010) and applied to the echelle spectral measurements of the HerbigHaro object HH 202-S in the Orion Nebula, both for the diffuse and shocked high-density gas (Mesa-Delgado et al. 2009). With the new atomic data, the agreement between the model and observation is much improved.

In addition to many others (c.f. viewgraphs of the presentation for an incomplete list of papers published in the last five years), six papers have been published by the Iron Project (e.g. Del Zanna, Storey \& Mason 2010; Storey \& Zeippen 2010), presenting radiative transition probabilities and collision strengths for many iron-peak elements.

\section{Neutron-capture elements}

With the now available large aperture telescopes equipped with high throughput and high spectral resolution spectrograph, very deep spectra can now be obtained for some of the brightest PNe in an affordable amount of observing time, allowing detections and measurements of lines as faint as one millionth of $\mathrm{H} \beta$ (Fang \& Liu 2011; Szyszka et al. this volume). In addition to a host of permitted transitions from ions of the abundant second- and third-row elements, many of them are of recombination origin, CELs from ions of extremely low abundance elements have been detected, such as fluorine (Zhang \& Liu 2005) and many neutron-capture elements (Sharpee et al. 2007). The abundances of those trace elements contain a wealth of information concerning the evolution and nucleosynthesis of low- and intermediate-mass stars (Karakas et al. 2009, and in this volume). One of the main obstacle hindering full usage of this gold mine of information has been the lack of atomic data for neutron-capture elements, notably those needed to solve for their ionization equilibrium (and hence to convert the measured ionic abundances to 
elemental abundances). To meet the need, Sterling \& Witthoeft (2011) and Sterling (2011) have recently presented multi-configuration Breit-Pauli distorted-wave photoionization cross-sections and radiative and di-electronic recombination rate coefficients for the first six ions of the trans-iron elements selenium and krypton, respectively. Charge exchange reaction rate coefficients for low-charge $\mathrm{Ge}, \mathrm{Se}, \mathrm{Br}, \mathrm{Kr}, \mathrm{Rb}$ and Xe ions reacting with neutral hydrogen are also obtained by Sterling \& Stancil (2011) adopting a multi-channel Landau Zener approach. The publication of these new atomic data will have a major impact on the investigations of neutron-capture element abundances and nucleosynthesis via nebular spectroscopy.

\section{He I recombination spectrum}

Helium is the second most abundant element after hydrogen. While the Balmer decrement and discontinuity near $3646 \AA$ of the $\mathrm{H}$ I recombination spectrum has long been used as an estimator of the interstellar reddening and diagnostics of electron temperature and density (Peimbert 1971; Barker 1978; Liu \& Danziger 1993; Liu et al. 2000), the diagnostic value of the recombination spectra of singly and doubly ionized helium has not received much attention until recently, possibly because of the observational difficulties, particularly in measuring the weak helium recombination continuum. Zhang et al. (2009) measure the $\mathrm{He}$ I discontinuity at $3421 \AA$, produced by $\mathrm{He}^{+}$recombination to the $\mathrm{He} \mathrm{I}$ $2 \mathrm{p}^{3} \mathrm{P}^{\mathrm{o}}$ level, in five PNe. The discontinuity is weak and hard to measure. Also in high excitation $\mathrm{PNe}$, the spectral region is plagued by strong emission lines including the [Ne $\mathrm{V}]$ $\lambda 3425$ line and the Bowen fluorescence lines O III $\lambda \lambda 3428,3444$, making accurate measurements all the more difficult. In spite of the large uncertainties, electron temperatures derived from the discontinuity are in general agreement with those deduced from He I recombination line ratios (Zhang et al. 2005), and are systematically lower than values yielded by the $\mathrm{H}$ i Balmer discontinuity, in line with the expectations of the bi-abundance model proposed by Liu et al. (2000).

Liu et al. $(2000,2001)$ find that for the singlet series of the He I recombination line spectrum, while the observed intensities of the $2 \mathrm{p}^{1} \mathrm{P}^{\mathrm{o}}-n \mathrm{~d}^{1} \mathrm{D}$ series are in good agreement with the predictions of the case $\mathrm{B}$ recombination theory, those of the $2 \mathrm{~s}^{1} \mathrm{~S}-n \mathrm{p}^{1} \mathrm{P}^{\mathrm{o}}$ and $2 \mathrm{p}^{1} \mathrm{P}^{\mathrm{o}}-n \mathrm{~s}^{1} \mathrm{~S}$ series are weaker by a factor of $2-3$ and 40 per cent, respectively. The results cannot be explained by self-absorption effects as in the case of the triplet lines. One possibility is that a significant fraction of the He I resonance line photons are destroyed either by photoionization of neutron hydrogen or by absorption of dust grains. Detailed photoionization modeling is required to verify these possibilities and quantify their effects on the observed line strengths.

\section{Heavy element optical recombination lines (ORLs)}

There is strong evidence that at least some PNe contain another previously unknown component of plasma, probably in the form of H-deficient (metal-rich) inclusions embedded in the diffuse gas of "normal" chemical composition (Liu 2006). Cooled by heavy element infrared fine-structure lines, the inclusions have very low temperatures $(\sim 1,000 \mathrm{~K})$ and thus do not contribute to the emission of optical/UV CELs, but are copious emitters of heavy element ORLs. Three-dimensional bi-abundance photoionization models incorporating H-deficient inclusions show that only a small amount of $\mathrm{H}$-deficient material, about one Jupiter mass, is required to explain the strengths of heavy element ORLs that are otherwise hard to reproduce with a chemically homogeneous model (Yuan et al. 2011). 

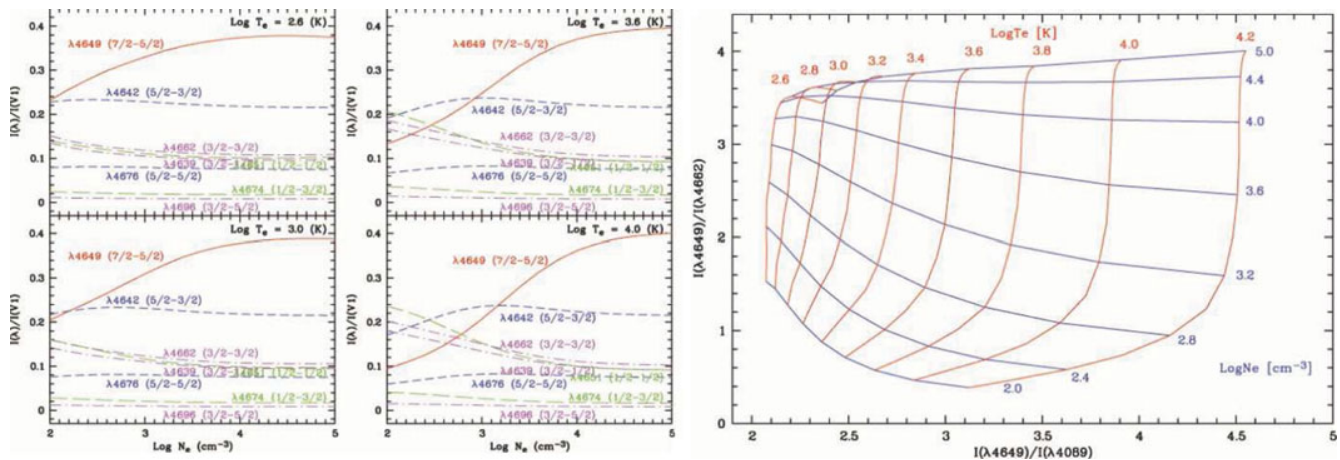

Figure 1. Left: Fractional intensities of the O II Multiplet V 1 as a function of electron density at four selected temperatures; Right: Loci of the O II $\lambda 4649 / \lambda 4662$ and $\lambda 4649 / \lambda 4089$ ratios for a grid of temperatures and densities. The plots are based on data communicated by Dr. P. J. Storey.

In order to betterconstrain the physical properties, chemical composition and possible origins of the postulated $\mathrm{H}$-deficient inclusions, it is necessary to have atomic data, most important of all, the effective recombination coefficients for heavy element ORLs, that are valid at the very low electron temperatures prevailing in the $\mathrm{H}$-deficient inclusions (a few hundred Kelvin), and to develop diagnostics that allow one to probe electron temperature and density and elemental abundances of the H-deficient inclusions. At such low temperatures, di-electronic recombination via the fine-structure autoionizing levels between the lowest ionization thresholds of the recombining ion becomes significant and thus needs to be incorporated in the calculations. In addition, earlier calculations of the effective recombination coefficients of heavy element ions implicitly assume that the fine-structure levels of the ground term of the recombining ion are thermally populated proportional to their statistical weights, an assumption that has been known to be inaccurate under low density nebular conditions (Liu 2003; Ruiz et al. 2003).

New ab initio calculations of the effective recombination coefficients, valid down to very low temperatures and taking into account the dependence of the level populations of the ground states of the recombining ion, are now available for the recombination spectrum of O II (Bastin \& Storey 2006; Storey P. J., in preparation) and N II (Fang, Storey \& Liu 2011). The four panels on the left of Fig. 1 show the variations of the fractional intensities of the $\mathrm{O}$ II Multiplet V $13 \mathrm{p}^{4} \mathrm{D}^{\mathrm{o}}-3 \mathrm{~s}{ }^{4} \mathrm{P}$ as a function of electron density at four selected temperatures. The intensity ratio of components $\lambda 4649(J=7 / 2-5 / 2)$ and $\lambda 4662(J=$ $3 / 2-3 / 2)$ is sensitive to electron density. The predicted fractional multiplet intensity of the $\lambda 4649$ component as a function density is in good agreement with the observations (Bastin \& Storey 2005). A good temperature diagnostics is provided by the ratio of the $\lambda 4649$ line of Multiplet V 1 and the $4 \mathrm{f} \mathrm{G}[5]_{11 / 2}^{\circ}-3 \mathrm{~d}^{4} \mathrm{~F}_{9 / 2} \lambda 4089$ line, the strongest from the $4 \mathrm{f}-3 \mathrm{~d}$ configuration. The right panel of Fig. 1 shows the loci of the $\lambda 4649 / \lambda 4662$ and $\lambda 4649 / \lambda 4089$ ratios for a grid of temperatures and densities. Similar diagnostics have also been developed based on the N II recombination spectrum. In the case of $\mathrm{N}$ II, the intensity ratio of components $\lambda 5679(J=3-2)$ and $\lambda 5666(J=2-1)$ of Multiplet V 3 $3 \mathrm{p}^{3} \mathrm{D}-3 \mathrm{~s}^{3} \mathrm{P}^{\mathrm{o}}$ is a sensitive density diagnostic, whereas the ratio of the $\lambda 5679$ line and the $4 \mathrm{f} \mathrm{G}[9 / 2]_{5}-3 \mathrm{~d}^{3} \mathrm{~F}_{4}^{\mathrm{o}} \lambda 4041$ line, again the strongest from the $4 \mathrm{f}-3 \mathrm{~d}$ configuration, is sensitive to temperature (Fang, Storey \& Liu 2011).

With those new powerful plasma diagnostic tools based entirely on heavy element recombination lines, given high quality measurements, it is now possible to obtain accurate estimates of the (average) temperature and density under which the heavy element ORLs 

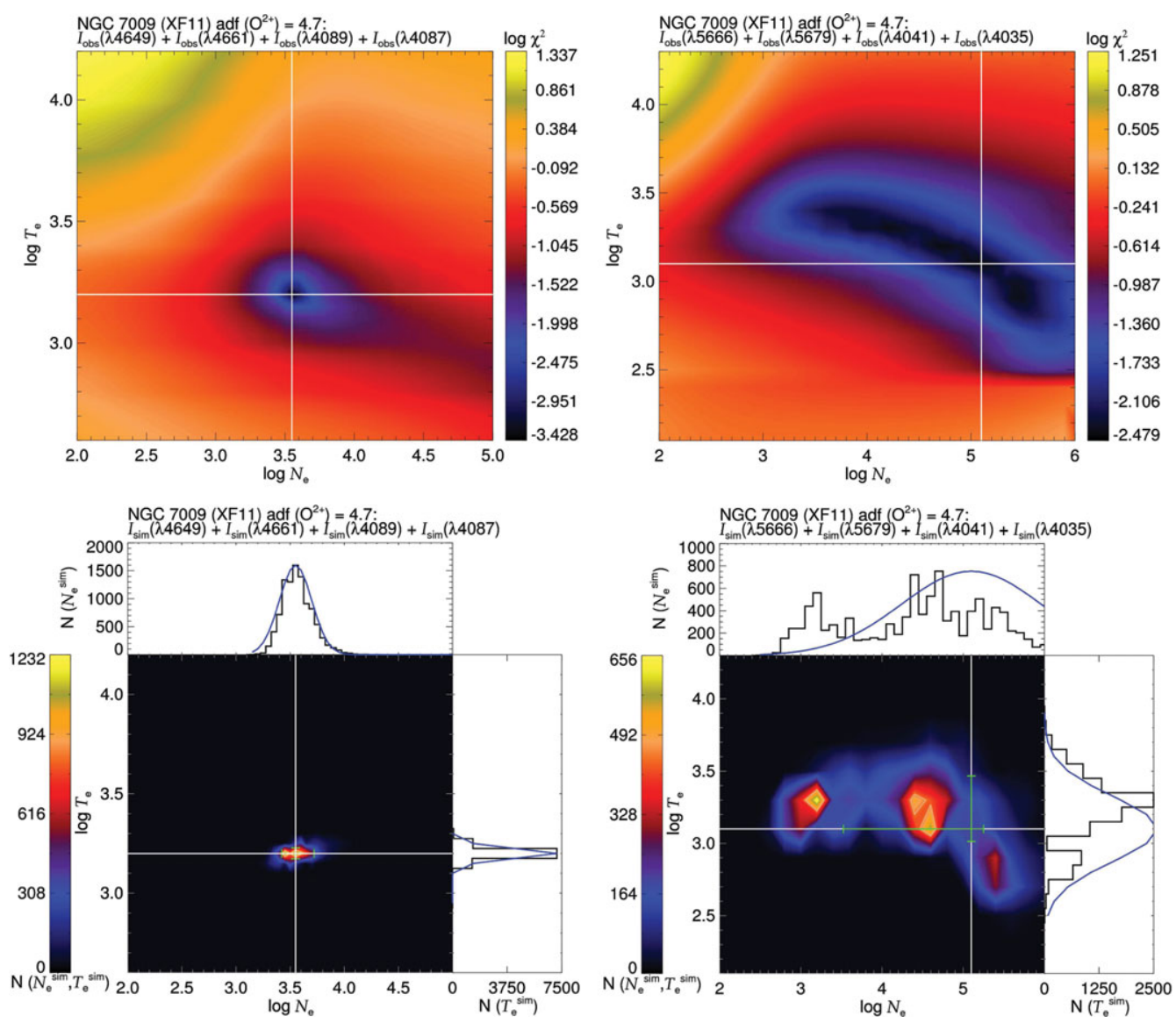

Figure 2. The distributions of $\log \chi^{2}\left(T_{\mathrm{e}}, N_{\mathrm{e}}\right)$ for a combination of four major O II (top-left) and N II (top-right) ORLs for the Saturn Nebula NGC 7009, with the measured line intensities given by Fang \& Liu (2011). The white crosshairs pinpoint the absolute minima. Assuming that the measured line intensities have a Gaussian error distribution, the distributions of the optimal $T_{\mathrm{e}}$ and $N_{\mathrm{e}}$ deduced from 10,000 realizations of the observations are given in the lower two panels. Again, the white crosshairs pinpoint the absolute minima yielded by the measured line intensities (McNabb et al., this volume and in preparation).

originate. Both $\mathrm{O}$ II and $\mathrm{N}$ II exhibit a rich recombination spectrum, with tens or even hundreds of lines falling in the optical. Rather than using some specific line ratios to estimate the density and/or temperature as shown in Fig. 1, ideally one could use all the detectable $\mathrm{O}$ II (or N II) ORLs simultaneously to constrain the temperature and density. In practice, most ORLs are faint and often blended with other lines. Thus successful application of those diagnostics requires not only high quality data, but also careful spectral identification and measurement to single out "good" lines that are least affected by line blending effects.

As an example, Fig. 2 shows the distributions of $\log \chi^{2}\left(T_{e}, N_{e}\right)$ for the Saturn Nebula NGC 7009, with the measured line intensities given by Liu \& Fang (2011), in fitting respectively a combination of four major $\mathrm{O}$ II and $\mathrm{N}$ II ORLs. The optimal values of $T_{\mathrm{e}}$ and $N_{\mathrm{e}}$ at the position of the absolute minimum of the $\chi^{2}$ distribution as yielded by the observed intensities are marked with a crosshair. If one assumes that the observed line intensities have a Gaussian error distribution, then the distributions of the optimal values of $T_{\mathrm{e}}$ and $N_{\mathrm{e}}$ yielded by 10,000 realizations of the observations are given in the 


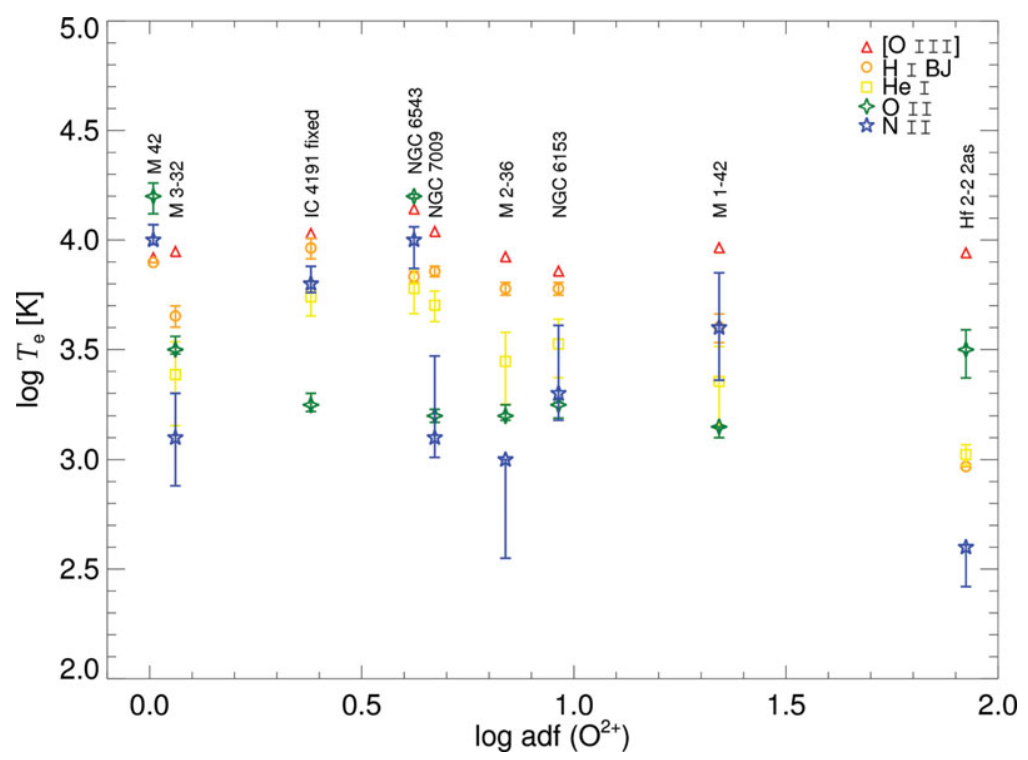

Figure 3. Comparison of electron temperatures derived respectively from the [O III] forbidden line ratio (triangles), the $\mathrm{H}$ I Balmer jump (circles), He I recombination line ratios (squares), and from O II (4-pointed stars) and N II ORLs (5-pointed stars), for the H II region the Orion Nebula (M 42) and a sample of PNe exhibiting different abundance discrepancy factor, $\operatorname{adf}\left(\mathrm{O}^{2+}\right)$, defined as the ratio of the $\mathrm{O}^{2+} / \mathrm{H}^{+}$ionic abundances derived from $[\mathrm{O}$ III] forbidden lines and from O II ORLs.

lower two panels of the Figure ( $\mathrm{McNabb}$ et al., this volume and in preparation). Both $T_{\mathrm{e}}$ and $N_{\mathrm{e}}$ are well constrained by the four O II ORLs adopted in the optimization, with a clean and almost Gaussian distribution of uncertainties. By contrast, in the case of $\mathrm{N}$ II, while $T_{\mathrm{e}}$ is still reasonably well constrained by the four adopted lines, $N_{\mathrm{e}}$ is poorly constrained. Furthermore, the distribution of the optimal values of $T_{\mathrm{e}}$ and $N_{\mathrm{e}}$ deduced from 10,000 realizations of the observations also exhibits multiple peaks, suggesting that small uncertainties in the measured line intensities can lead to large errors in the deduced values of $T_{\mathrm{e}}$ and $N_{\mathrm{e}}$. In addition, $\mathrm{N}$ II has a ground state of $2 \mathrm{p}^{2}{ }^{3} \mathrm{P}$, thus multiplets originating from spectral terms of low total angular momenta, such as V $33 \mathrm{p}^{3} \mathrm{D}-3 \mathrm{~s}^{3} 3 \mathrm{P}^{\mathrm{o}}$, are susceptible to contaminations from fluorescence excitation (Grandi 1976). However, $\mathrm{N}$ II ORLs originating from spectral terms of large total angular momenta, including the $4 \mathrm{f} \mathrm{G}[9 / 2]_{5}-3 \mathrm{~d}^{3} \mathrm{~F}_{4}^{\mathrm{o}} \lambda 4041$ line discussed above, and all singlet multiplets are unlikely contaminated. O II has a ground state $2 \mathrm{p}^{3}{ }^{4} \mathrm{~S}^{\circ}$, thus most $\mathrm{O}$ II ORLs, including those discussed above, are unlikely to be affected by fluorescence excitation.

Fig. 3 compares electron temperatures derived from various diagnostics for a sample of objects exhibiting different value of $\operatorname{adf}\left(\mathrm{O}^{2+}\right)$, defined as the ratio of the $\mathrm{O}^{2+} / \mathrm{H}^{+}$ ionic abundances derived from collisional excited [O III] forbidden lines and from $\mathrm{O}$ II ORLs. In Hf 2-2, the object having the largest value of adf found so far, all recombination line or continuum diagnostics, including the $\mathrm{H}$ I Balmer jump and He I, O II and N II ORLs, yield consistent temperatures of around 1,000 K, almost an order of magnitude lower than the [O III] forbidden line temperature. For other PNe showing lower but still significant adf values, O II ORLs yield $T_{\mathrm{e}}$ 's around 1,000 K. Similar results are found from $\mathrm{N}$ II ORLs, but with larger uncertainties, probably caused by measurement uncertainties, or by contaminations of excitation mechanisms other than recombination as discussed above. The Balmer jump of the $\mathrm{H}$ I recombination continuum or He I recombination line 
ratios yield values of $T_{\mathrm{e}}$ generally falling between the values deduced from collisionally excited [O III] forbidden lines and those derived from $\mathrm{O}$ II or N II ORLs, suggesting that line or continuum emission of $\mathrm{H} \mathrm{I}$ and $\mathrm{He} \mathrm{I}$ is only partially affected by the presence of low-temperature H-deficient inclusions. It is assuring that in the Orion Nebula (M 42), an $\mathrm{H}$ II region exhibiting negligible abundance discrepancy (adf $\sim 1$ ), all diagnostics, forbidden lines or recombination lines/continua likewise, yield consistent temperatures around $10,000 \mathrm{~K}$.

The very low values of $T_{\mathrm{e}}$ around $1,000 \mathrm{~K}$ yielded by $\mathrm{O}$ II and $\mathrm{N}$ II ORLs, deduced based on the new high quality atomic data, are about an order of magnitude lower than the forbidden line temperatures. The result provides the strongest direct evidence that heavy element ORLs in PNe in general, or at least in those exhibiting large adf values, originate from low temperature H-deficient inclusions, in support of the bi-abundance model proposed by Liu et al. (2000).

\section{Acknowledgments}

I thank Dr. Xuan Fang and Mr. Ian McNabb for their assistance in the preparation of this contribution. The work is partially supported by NSFC Grant \#10933001.

\section{References}

Barker, T. 1978, ApJ, 219, 914

Bastin, R. \& Storey, P. J. 2005, in: R. Szczerba, G. Stasinska \& S. K. Gorny (eds.), Planetary Nebulae as Astronomical Tools (Springer, New York), AIP Conf. Proc., Vol. 804, p.63

Bastin, R. \& Storey, P. J. 2006, in: M. J. Barlow and R. H. Méndez (eds.), Planetary Nebulae in our Galaxy and Beyond (Cambridge: Cambridge University Press), p. 369

Bautista, M. A. 2006, in: M. J. Barlow and R. H. Méndez (eds.), Planetary Nebulae in our Galaxy and Beyond (Cambridge: Cambridge University Press), p. 203

Bautista, M. A., Ballance, C. P., \& Quinet, P. 2010, ApJ, 718, L189

Chen, S. H., Qing, B., \& Li, J. M. 2007, Phys. Rev. A, 76, 042507

Del Zanna, G., Storey, P. J., \& Mason, H. E. 2010, A\&A, 514, 40

Fang, X. \& Liu, X. W. 2011, MNRAS, 415, 181

Fang, X., Storey, P. J., \& Liu, X. W. 2011, A\&A, 530, 18

Grandi, S. A. 1976, ApJ, 206, 658

Karakas, A. I., van Raai, M. A., Lugaro, M., Sterling, N. C., \& Dinerstein, H. L. 2009, ApJ, 690,1130

Liu, X. W. 2003, in: S. Kwok, M. Dopita \& R. Sutherland (eds.), Planetary Nebulae: Their Evolution and Role in the Universe (San Francisco: ASP), p.339

Liu, X. W. 2006, in: M. J. Barlow and R. H. Méndez (eds.), Planetary Nebulae in our Galaxy and Beyond (Cambridge: Cambridge University Press), p. 219

Liu, X. W. \& Danziger, I. J. 1993, MNRAS, 263, 256

Liu, X. W., Luo, S.-G., Barlow, M. J., Danziger, I. J., \& Storey, P. J. 2001, MNRAS, 327, 141

Liu, X. W., Storey, P. J., Barlow, M. J., Danziger, I. J., Cohen, M., \& Bryce, M. 2000, MNRAS, 312,585

Mesa-Delgado, A., Esteban, C., García-Rojas, J., Luridiana, V., Bautista, M., Rodríguez, M., López-Martín, L., \& Peimbert, M. 2009, MNRAS, 395, 855

Nahar, S. N. 2003, in: S. Kwok, M. Dopita \& R. Sutherland (eds.), Planetary Nebulae: Their Evolution and Role in the Universe (San Francisco: ASP), p.325

Peimbert, M. 1971, Bol. Obs. Tonantzintla Tacubaya, 6, 29

Péquignot, D. 2008, A\&SA, 478, 371

Ruiz, M. T., Peimbert, A., Peimbert, M., \& Esteban, C. 2003, ApJ, 595, 247

Sharpee, B., Zhang, Y., Williams, R., Pellegrini, E., Cavagnolo, K., Baldwin, J. A., Phillips, M., \& Liu, X. W. 2007, ApJ, 659, 1265 
Sterling, N. C. 2011, $A \mathscr{E} A$ A, 533, 62

Sterling, N. C. \& Stancil, P. C. 2011, A\&A, 535, A117

Sterling, N. C. \& Witthoeft, M. C. 2011, $A \& A$, 529, 147

Storey, P. J. \& Zeippen, C. J. 2010, A\&A, 511, 78

Tayal, S. S. 2007, ApJS, 171, 331

Tayal, S. S. 2011, ApJS, 195, 11

Tayal, S. S. \& Zatsarinny, O. 2010, ApJS, 188, 32

Yuan, H.-B., Liu, X. W., Péquignot, D., Rubin, R. H., Ercolano, B., \& Zhang, Y. 2011, MNRAS, 411,1035

Zhang, Y. \& Liu, X. W. 2002, MNRAS, 337, 499

Zhang, Y. \& Liu, X. W. 2003, A\&A, 404, 545

Zhang, Y. \& Liu, X. W. 2005, ApJ, 631, L61

Zhang, Y., Liu, X. W., Liu, Y., \& Rubin, R. H. 2005, MNRAS, 358, 457

Zhang, Y., Yuan, H. B., Hua, T. T., Liu, X. W., Nakashima, J., \& Kwok, S. 2009, ApJ, 695, 488 